\title{
Phenolic Compound Profiles and their Corresponding Antioxidant Capacity of Purple Pitaya (Hylocereus sp.) Genotypes
}

\author{
Patricia Esquivel $^{\mathrm{a}, \mathrm{b}}$, Florian C. Stintzing ${ }^{\mathrm{a}, \mathrm{c}, *}$, and Reinhold Carle ${ }^{\mathrm{a}}$ \\ ${ }^{a}$ Institute of Food Science and Biotechnology, Section Plant Foodstuff Technology, \\ Hohenheim University, August-von-Hartmann-Straße 3, D-70599 Stuttgart, Germany \\ b Escuela de Tecnología de Alimentos, Universidad de Costa Rica, 2060 San Pedro, \\ Costa Rica \\ c Present address: WALA Heilmittel GmbH, Dorfstraße 3, D-73087 Bad Boll/Eckwälden, \\ Germany. E-mail: florian.stintzing@wala.de \\ * Author for correspondence and reprint requests \\ Z. Naturforsch. 62 c, 636-644 (2007); received February 15/March 13, 2007
}

\begin{abstract}
Folin-Ciocalteu and TEAC (Trolox equivalent antioxidant capacity) assay together with the spectrophotometric determination of betalains were applied to investigate the correlation between phenolics and their contribution to the antioxidant capacity of five different Costa Rican genotypes of purple pitaya (Hylocereus sp.) and of H. polyrhizus fruits. Maximum antioxidant capacity, total phenolic and betalain contents were observed in the genotype 'Lisa'. While non-betalainic phenolic compounds contributed only to a minor extent, betalains were responsible for the major antioxidant capacity of purple pitaya juices evaluated. The phenolic pattern of each genotype was also thoroughly investigated using liquid chromatography coupled to positive electrospray ionization (ESI) tandem mass spectrometry. In addition to the well known betalains previously reported in Hylocereus fruits, several biosynthetic precursors were detected. Notably, decarboxylated and dehydrogenated betalains were identified as genuine compounds of the juices. Some of these compounds were previously described as artifacts upon heat exposure. Moreover, gallic acid was identified for the first time in pitaya fruits. While the phenolic profiles generally differed between genotypes, phenolic compound composition of 'Rosa' resembled that of $H$. polyrhizus with respect to total contents of betacyanins, betalainic precursors, phyllocactin and cyclo-Dopa malonyl-glucosides.
\end{abstract}

Key words: Antioxidant Capacity, Betalains, Phenolics

\section{Introduction}

Vine cacti, native to tropical regions of North, Central and South America, are known in Latin America as pitahaya or pitaya. The plant has three-ribbed stems that climb to trees and rocks. The fruit is a medium- to large-size epygenous berry characterized by large leaf-like scales (Nerd and Mizrahi, 1997). Selected species have been recently developed as fruit crops. Among them, $\mathrm{Hy}$ locereus undatus [(Haworth) Britton \& Rose] (red skin, white pulp) has been widely planted, while others such as $H$. polyrhizus [(F. A. C. Weber) Britton \& Rose] (red skin, red-violet pulp) and H. costaricensis [(F. A. C. Weber) Britton \& Rose] (red skin, red pulp) are grown at a smaller scale (Mizrahi and Nerd, 1999). 'Lisa', 'Orejona', 'Rosa' and 'San Ignacio' are common genotypes commercialized in Nicaragua, the Central American country with the highest pitaya production of about
3000 t on 420 ha (Anonymous, 1994; Vaillant et al., 2005), while the genotype 'Nacional' is grown in the region of Puntarenas, Costa Rica. The red pitayas, rich in betalains, have received scientific significance only recently due to their potential as a source of natural food pigments (Stintzing et al., 2002), but also because of their antioxidant capacity (Vaillant et al., 2005). Wu et al. (2006) studied the total phenolic content, and antioxidant and antiproliferative activity of red pitaya on melanoma cells to evaluate its pharmacological potential. According to their work, flesh and peel were both rich in phenolics and good sources of antioxidants. These findings were confirmed by Vaillant et al. (2005) who found $560-680 \mu \mathrm{mol}$ gallic acid equivalents resulting in ORAC values of $880-$ $1130 \mu \mathrm{mol}$ equivalents of Trolox per $100 \mathrm{~g}$ fresh fruit, where Trolox represents a water-soluble vitamin $\mathrm{E}$ analogue commonly applied to express radi- 
cal scavenging capacity equivalents of various substances or extracts (Sánchez-Moreno, 2002). In addition, several studies have shown that betalains are efficient free radical scavengers preventing oxygen-induced and free radical-mediated oxidation of biological molecules (Kanner et al., 2001; Butera et al., 2002; Stintzing et al., 2005).

Although high antioxidant levels have been reported in pitayas, studies on the phenolic profile of Hylocereus fruits are still lacking. In a very recent work by Mahattanatawee et al. (2006), the phenolic fraction was assumed to be mainly composed of hydroxycinnamic acids, however, these authors did not provide further evidence. Knowledge on the phenolic composition of Hylocereus juices is of importance, considering that phenolics are antioxidants and may inhibit specific interactions with cell receptors and enzymes depending on their particular chemical structure. Therefore, the present work aimed at a thorough investigation of the phenolic patterns of pitaya fruits using liquid chromatography coupled to positive electrospray ionization (ESI) tandem mass spectrometry. In addition, total betalains and total phenolics should be assessed including their respective contribution to the antioxidant capacity of pitaya fruit juices applying the TEAC assay.

\section{Materials and Methods}

\section{Plant material and sample preparation}

Three-year-old plants of the genotypes 'Lisa', 'Rosa', 'San Ignacio', 'Orejona' and 'Nacional', cultivated organically in Barranca, Puntarenas, Costa Rica (N 9 57.566', W 84 43.217') were used for this study. Samples of each of the analyzed genotypes were deposited at the Herbarium of the Universidad de Costa Rica (USJ), where they received the following accession numbers: 'Rosa' 88680, 'San Ignacio' 88681, 'Nacional' 88682, 'Lisa' 88683 and 'Orejona' 88684. Three fruits of each genotype were harvested from July to August 2005, when their peel colour started to turn from green to purple. Fruits were stored at room temperature until the purple colour developed completely and were subsequently frozen at $-20{ }^{\circ} \mathrm{C}$ until analysis. After thawing, the fruits were peeled and the pulp was manually pressed. The juices were filtered using a filter paper (520 $\mu \mathrm{m}$, Schleicher-Schuell, Dassel, Germany). For comparative purposes, a sample of $H$. polyrhi- zus brought from Israel in 2005 and cultivated under greenhouse conditions was also analyzed.

\section{Solvents and reagents}

Reagents and solvents were purchased from VWR International (Darmstadt, Germany) and were of analytical or HPLC grade. Gallic acid and tyrosine were obtained from Sigma-Aldrich $\mathrm{GmbH}$ (Steinheim, Germany). Deionized water was used throughout.

\section{Total betalain content}

Photometric quantification of total betalains was carried out in duplicate following the method by Kugler et al. (2007) using a UV-vis spectrophotometer (Perkin-Elmer, Überlingen, Germany) equipped with UVWinLab V 2.85.04 software (Perkin-Elmer Instruments, Norwalk, CT, USA).

\section{Total phenolic contents}

Total phenolic compounds were assessed photometrically applying the Folin-Ciocalteu assay (Singleton et al., 1999). Absorption was registered at $720 \mathrm{~nm}$ using a UV-vis spectrophotometer (Perkin-Elmer) equipped with UVWinLab V 2.85.04 software (Perkin-Elmer Instruments). All determinations were performed in duplicate. A fourpoint calibration curve was used for quantification of total phenolic contents, which were expressed as ascorbic acid equivalents (AAE). The particular contribution of betacyanins and ascorbic acid to the AAE values of total phenolic contents as determined by the Folin-Ciocalteu assay was assessed using standard curves for both compounds and phenolic values were corrected accordingly. For this purpose, ascorbic acid concentrations were determined following a method by Esquivel et al. (2007), whereas betanin purification, isolation and quantification were conducted as described elsewhere (Stintzing et al., 2004).

\section{Antioxidant capacity}

The photometric TEAC assay, the reduction of the in vitro induced 2,2-azinobis-(3-ethylbenzothiazoline-6-sulfonic acid) diammonium salt (ABTS) radical described by Van den Berg et al. (2000), was slightly modified: ABTS was dissolved in a $\mathrm{pH}$ 7.4 phosphate buffer medium containing $\mathrm{NaCl}$ at a concentration of $20 \mathrm{~mm}$. A volume of $0.5 \mathrm{~mL}$ of this reagent was added to $100 \mathrm{~mL}$ of a solution of 2,2'-azobis-(2-amidinopropane)- $\mathrm{HCl}$ (ABAP) in 
phosphate buffer $(2.5 \mathrm{~mm})$ and subsequently incubated during $15 \mathrm{~min}$ at $60{ }^{\circ} \mathrm{C}$ in the dark. $40 \mu \mathrm{L}$ of the diluted sample were added to $2 \mathrm{~mL}$ of the ABTS radical solution and mixed thoroughly. After a reaction time of $6 \mathrm{~min}$, absorbance was assessed at $734 \mathrm{~nm}$. All measurements were performed in duplicate, and for each sample a freshly pipetted blank was measured. A standard solution of ascorbic acid was used for calibration and the results were expressed as AAE, according to Chun et al. (2005).

\section{Phenolic compound profile}

The phenolic patterns of the juices were studied using an HPLC-system (Merck, Darmstadt, Germany) equipped with an analytical Sunfire $\mathrm{C}_{18^{-}}$ column $(250 \times 4.6 \mathrm{~mm}$ i. d., Waters, Wexford, Ireland) with a particle size of $5 \mu \mathrm{m}$. Separation was achieved at $30^{\circ} \mathrm{C}$ applying a flow rate of $1 \mathrm{~mL}$ $\min ^{-1}$. The mobile phase A consisted of $5 \%(\mathrm{v} / \mathrm{v})$ formic acid in water, while $\mathrm{MeCN} 100 \%$ was used as the mobile phase $\mathrm{B}$. The first $5 \mathrm{~min}$ were performed isocratically with $100 \%$ A. Then, a linear gradient was followed to $5 \% \mathrm{~B}$ at $35 \mathrm{~min}, 12 \% \mathrm{~B}$ at $42 \mathrm{~min}, 30 \% \mathrm{~B}$ at $55 \mathrm{~min}$ and finally $100 \% \mathrm{~B}$ at $70 \mathrm{~min}$, before re-equilibration to starting conditions. Monitoring was carried out at $280 \mathrm{~nm}$. Relative chromatogram areas for individual peaks were calculated. All determinations were performed in duplicate.

\section{Assignment of betalains and other compounds by $H P L C-D A D-M S^{2}$}

Applying identical chromatographic conditions, identification of compounds was achieved by
HPLC-MS analyses on a series 1100 HPLC instrument (Agilent, Waldbronn, Germany), equipped with ChemStation software, a G1322A degasser, a G1312A binary gradient pump, a G1329/1330A autosampler, a G1316A column-oven, and a G1315A diode array detector. The HPLC system was connected in series with a Bruker model Esquire 3000+ (Bremen, Germany) ion trap mass spectrometer fitted with an electrospray ionization source operating in the positive mode. Nitrogen was used as dry gas at a flow rate of $12 \mathrm{~mL} \mathrm{~min}^{-1}$ and a pressure of $70 \mathrm{psi}$. The nebulizer temperature was set to $365^{\circ} \mathrm{C}$. Using helium as collision gas $\left(4.1 \times 10^{-9}\right.$ bar $)$, collision-induced dissociation spectra were obtained with a fragmentation amplitude of $1.2 \mathrm{~V}$ (MS/MS).

\section{Statistical analysis}

To determine differences between genotypes, analysis of variance and mean comparisons using the Tukey test were performed (Statistica for Windows, StatSoft, Tulsa, OK, USA).

\section{Results and Discussion}

\section{Total contents of betalains and colourless phenolics}

The betalain contents of juices from different Hylocereus genotypes are shown in Table I. Differences were observed among the genotypes, with 'Lisa' exhibiting the highest and 'San Ignacio' and 'Rosa' the lowest pigment contents. Total phenolic contents of the juices were measured with the Folin-Ciocalteu method (Table I). Apart from phenolic compounds, other easily oxidizable substan-

Table I. Total betacyanins, total phenolics and antioxidant capacity (TEAC) values of different Hylocereus genotypes ${ }^{\mathrm{a}}$.

\begin{tabular}{|c|c|c|c|c|c|}
\hline Genotype & $\begin{array}{c}\text { Total } \\
\text { betalains } \\
{[\mathrm{mg} / 100 \mathrm{~mL}]}\end{array}$ & $\begin{array}{l}\text { Total phenolics }{ }^{\mathrm{b}, \mathrm{c}} \\
\text { Folin-Ciocalteu } \\
\text { [mg/100 mL] }\end{array}$ & $\begin{array}{c}\text { Betalains }^{\mathrm{d}} \\
\text { Folin-Ciocalteu }^{(\%)}\end{array}$ & 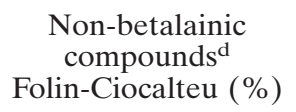 & $\begin{array}{c}\mathrm{TEAC}^{\mathrm{b}, \mathrm{c}}(\mathrm{AAE}) \\
{[\mathrm{mg} / 100 \mathrm{~mL}]}\end{array}$ \\
\hline 'Lisa' & $75.1 \pm 3.9 \mathrm{~A}$ & $13.3 \pm 0.4 \mathrm{~A}$ & $79.9 \pm 0.6 \mathrm{C}$ & $20.1 \pm 0.6 \mathrm{~B}$ & $36.1 \pm 3.4 \mathrm{~A}$ \\
\hline 'Nacional' & $67.4 \pm 1.6 \mathrm{~B}$ & $10.0 \pm 0.5 \mathrm{C}$ & $76.1 \pm 1.2 \mathrm{D}$ & $23.9 \pm 1.2 \mathrm{~A}$ & $34.8 \pm 1.5 \mathrm{~A}$ \\
\hline 'Orejona' & $62.1 \pm 1.0 \mathrm{C}$ & $11.2 \pm 0.1 \mathrm{~B}$ & $80.4 \pm 0.2 \mathrm{C}$ & $19.6 \pm 0.2 \mathrm{~B}$ & $27.9 \pm 0.7 \mathrm{CB}$ \\
\hline 'Rosa' & $43.2 \pm 0.6 \mathrm{E}$ & $12.9 \pm 0.2 \mathrm{~A}$ & $88.1 \pm 0.2 \mathrm{~A}$ & $11.9 \pm 0.2 \mathrm{D}$ & $26.8 \pm 1.3 \mathrm{~B}$ \\
\hline 'San Ignacio' & $43.2 \pm 0.6 \mathrm{E}$ & $9.6 \pm 0.7 \mathrm{C}$ & $84.0 \pm 0.1 \mathrm{~B}$ & $16.0 \pm 0.1 \mathrm{C}$ & $24.5 \pm 0.8 B$ \\
\hline H. polyrhizus & $57.1 \pm 0.5 \mathrm{D}$ & $9.2 \pm 0.1 \mathrm{C}$ & $78.1 \pm 0.3 \mathrm{CD}$ & $21.9 \pm 0.3 \mathrm{AB}$ & $30.5 \pm 0.1 \mathrm{~A}$ \\
\hline
\end{tabular}

a Significant differences within values in the same column are indicated by different letters $(P<0.05)$.

$\mathrm{b}$ Ascorbic acid equivalents in $\mathrm{mg} / 100 \mathrm{~mL}$.

${ }^{c}$ Since ascorbic acid was not detected in the juices, no correction was required.

d Based on calculated values of the respective total betacyanin content. 
ces such as ascorbic acid are also determined by the Folin-Ciocalteu reagent, producing a blue colour following the reduction of yellow heteropolyphosphomolybdate-tungstate anions. The maximum total phenolic content was observed in 'Lisa' and 'Rosa', while 'Nacional', 'San Ignacio' and $H$. polyrhizus marked the minimum values. Since ascorbic acid was only present in low amounts, complying with previous reports (Stintzing et al., 2003; Vaillant et al., 2005), its contribution to total phenolics was negligible. However, betalains substantially contributed to Folin-Ciocalteu values (Table I), ranging between $76.1 \%$ for 'Nacional' and $88.1 \%$ for 'Rosa'. This is in accordance with the results of Kujala et al. (2002) who evidenced a correlation between total betalains and total phenolics in beetroot peel extracts. As can be seen from Table I non-betalainic compounds contributed to Folin-Ciocalteu values to a much smaller extent $(11.9-23.9 \%)$.

\section{Antioxidant capacity}

The antioxidant capacity values (TEAC) for the juices of the different Hylocereus genotypes revealed significant differences among the genotypes (Table I). While the maximum TEAC value was found in 'Lisa', 'Nacional' and H. polyrhizus, lower values were assessed for the remaining genotypes. A positive correlation to the total betalain contents was observed $\left(R^{2}=0.75\right)$. Comparing these values with those of a previous investigation (Chun et al., 2005), pitaya fruits were in the same range with tomato, pumpkin and honeydew melon.

\section{Individual phenolic compounds in purple pitaya (Hylocereus sp.) fruits}

Individual phenolics of the juices from Hylocereus fruits are shown in Table II. The presence of gallic acid (1) and tyrosine (3) was confirmed by co-injection with commercially available standards and comparison with literature values (Sakakibara et al., 2003; Kammerer et al., 2004; Kugler et al., 2006).

Gallic acid was observed in all genotypes, with higher amounts in 'Rosa' and lowest in 'Orejona' and $H$. polyrhizus juices (Table II). Gallic acid is a well known natural antioxidant widely spread in plants (Wang et al., 2007) exhibiting anti-inflammatory, anti-fungal and anti-cancer properties (Cháfer et al., 2007). However, to the best of our knowledge, gallic acid has not been previously reported in Hylocereus fruits.
Since amines and amino acids together with betalamic acid are precursors in betalain biosynthesis, knowledge on the amino compound composition in betalainic fruits and vegetables is useful (Strack et al., 2003). Therefore, the findings of tyrosine representing a precursor of cyclo-Dopa is of particular interest (Table II).

Further phenolic compounds, such as ellagic acid (8) and acetylcoumarin (15), could only be tentatively assigned by their specific mass spectrometric data. Ellagic acid is common to fruits such as raspberries, strawberries, walnuts, grapes, and black currants (Rommel and Wrolstad, 1993; Priyadarsini et al., 2002), and taking into account the high reactivity of gallic acid, a derivative such as ellagic acid would be expected. Additionally, four unknown components $(\mathbf{5}, \mathbf{9}, \mathbf{1 1}, \mathbf{2 4})$ were detected. Considering their maximum absorbance and previous assumptions (Sakakibara et al., 2003; Mahattanatawee et al., 2006), these compounds are suspected to be hydroxycinnamic acid derivatives.

Nine different betalains were also detected at $280 \mathrm{~nm}$, namely trace amounts of the betaxanthin indicaxanthin (12), together with the betacyanins betanin (16), isobetanin (17), phyllocactin I (18), iso-phyllocactin I (19), phyllocactin II (20) descarboxy neobetanidin (21), decarboxylated neobetanin (22), as well as a neobetanidin structure being both dehydrogenated and decarboxylated (25). In addition, 5,5',6,6'-tetrahydroxy-3,3'-biindolyl (23) previously reported in red beet peel extracts was found. These compounds were assigned based on UV-vis and mass spectrometric data reported in previous studies (Kujala et al., 2001; Stintzing et al., 2002; Herbach et al., 2004, 2005; Wybraniec, 2005; Wybraniec and Mizrahi, 2005; Wybraniec et al., 2007).

The presence of components such as betalamic acid (10), 2-decarboxy-cyclo-Dopa (6), malonylglucoside (2), and cyclo-Dopa malonyl-glucosides $(4,7,13,14)$ may be explained by their involvement in betalain biosynthesis (Kobayashi et al., 2001). These compounds were identified by their UV-vis absorption maxima and mass spectrometric data in compliance with previous reports (Herbach et al., 2004, 2005). In accordance with a very recent study, where acyl migration was described for betacyanins acylated with malonic acid yielding phyllocactin I (6'-O-malonyl-betanin) and phyllocactin II (4'-O-malonyl-betanin) (Wybraniec et al., 2007), several compounds with spectrometric and mass spectrometric data matching those of cyclo- 


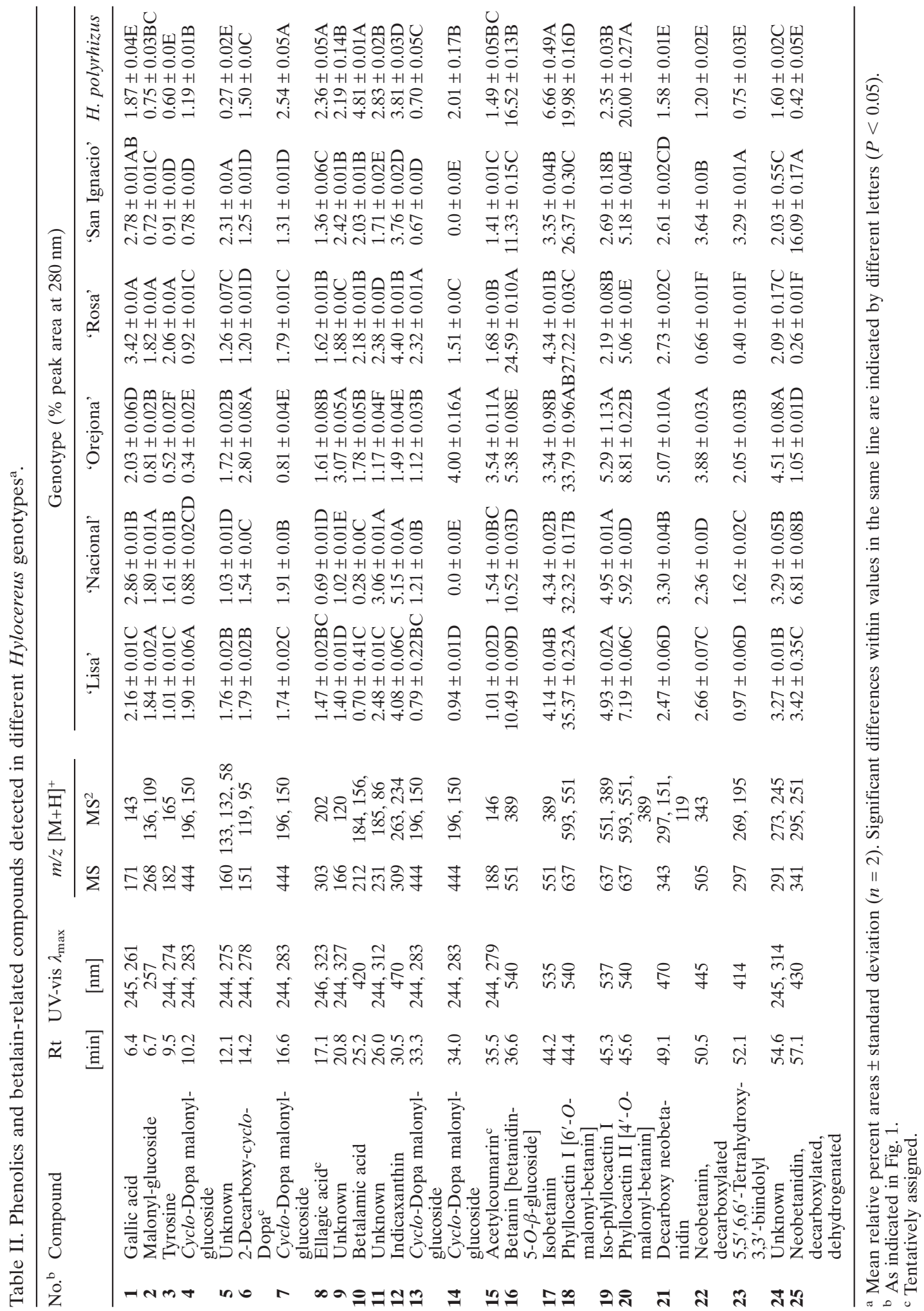




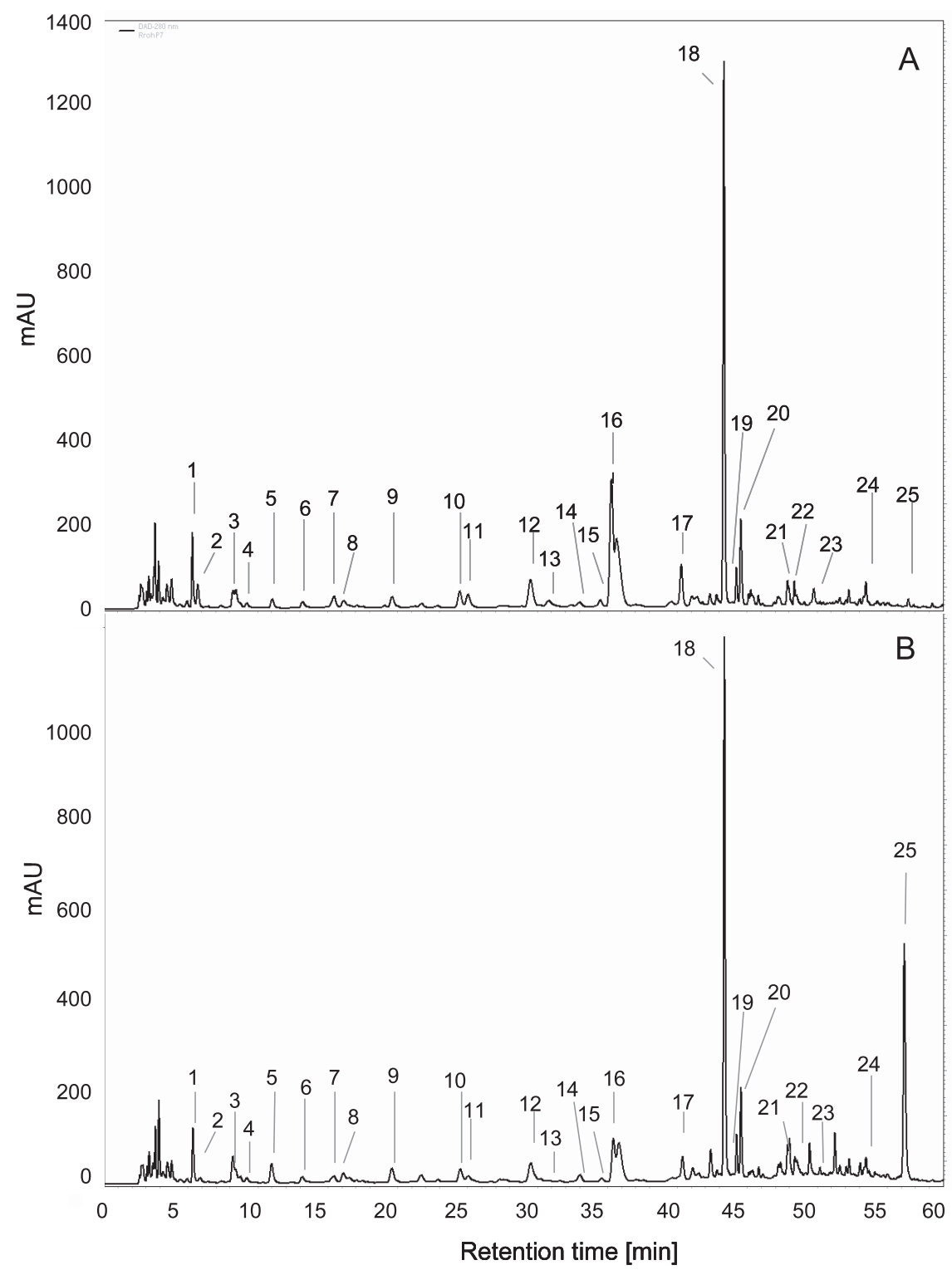

Fig. 1. Phenolic profile at $280 \mathrm{~nm}$ for the genotypes 'Rosa' (A) and 'San Ignacio' (B). Peak assignment is given in Table II.

Dopa malonyl-glucoside were detected. Being unstable when free, cyclo-Dopa was reported to react with glucose and betalamic acid, yielding cycloDopa glucoside and betanin and its $\mathrm{C}_{15}$-isomer isobetanin, respectively (Strack et al., 2003). Hence, the absence of this biosynthetic precursor in the juices is plausible. Accordingly, only trace amounts of cyclo-Dopa glucosides were detected in H. polyrhizus, 'San Ignacio', 'Rosa' and 'Lisa' (data not shown). Correspondingly, only traces of cyclo-Dopa-3-hydroxymethylglutaryl-glucoside, which is a hylocerenin precursor, were exclusively found in $H$. polyrhizus (data not shown).

Despite previous studies giving evidence of hylocerenin in the genus Hylocereus (Stintzing et al., 2002; Wybraniec and Mizrahi, 2005; Wybraniec et al., 2007), its occurrence could only be confirmed in $H$. polyrhizus where its co-elution 


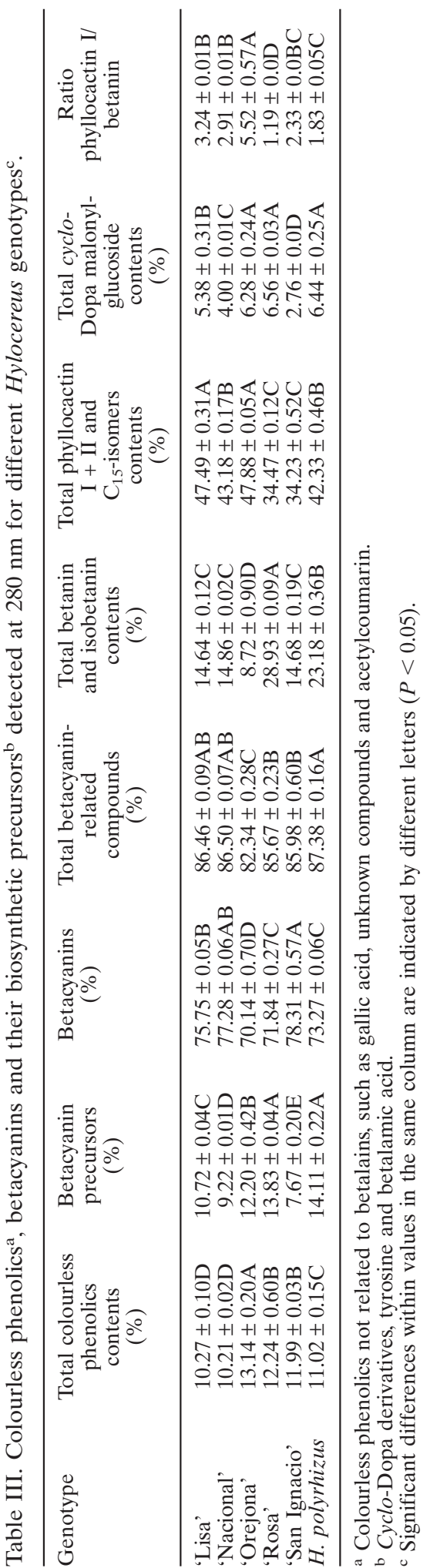

with iso-phyllocactin was observed (data not shown). Differences to earlier findings may be due to varying environmental conditions during cultivation.

Interestingly, decarboxylated and dehydrogenated betacyanins, previously described as degradation products of thermally treated Hylocereus polyrhizus fruit juices by Herbach et al. (2005) as well as Wybraniec and Mizrahi (2005), were found in all genotypes, although no heat treatment was applied in the present study. Therefore, our results indicate the inherent occurrence of both decarboxylating and dehydrogenating enzyme activities in Hylocereus fruits.

The individual chromatographic profiles of the different genotypes may allow to distinguish the genotypes (Table II). Representative chromatograms for 'Rosa' and 'San Ignacio' are shown in Fig. 1. Colourless phenolics unrelated to betalains ranged from $10 \%$ to $13 \%$ of the chromatogram area at $280 \mathrm{~nm}$ (Table III). With respect to total betacyanin-related compounds, significant differences among the genotypes were observed, ranging from 82 to $87 \%$. While $H$. polyrhizus was characterized by a high proportion of betacyanin precursors, amounting to $14.1 \%$, 'San Ignacio' exhibited the lowest ratio of $7.7 \%$ on a statistically significant basis (Table III). Moreover, the ratios between betalains and their precursors varied among the genotypes as can be seen from the sum of cyclo-Dopa malonyl-glucoside as compared to the corresponding total phyllocactin values. A useful marker for genotype differentiation appears to be the ratio of the predominant betacyanins betanin and phyllocactin I (Table III), except for 'Lisa' and 'Nacional' which seem to be closely related. The high proportion of betanin/isobetanin in 'Rosa' is of particular interest, because it parallels the antioxidant capacity values better than phyllocactin thus corroborating findings by Butera et al. (2002) postulating an important positive correlation between betanin contents and bioactivity (Tables I, III).

In summary, the individual phenolic compound profiles of six representatives of the genus Hylocereus were investigated for the first time. The antioxidant capacity of these fruits was found to be mainly based on betalains, followed by their biosynthetic precursors and finally by other non-betalainic phenolics, such as gallic acid and acetylcoumarin. Differences among the genotypes regarding their antioxidant activities were not only 
due to the respective betalain concentrations, but also determined by the individual betalain profiles. Furthermore, phenolic profiles among the genotypes differed significantly, with the genotype 'Rosa' resembling $H$. polyrhizus.

\section{Acknowledgements}

The authors wish to thank R. Crespo, F. BrenesAlvarez and E. Arias-Bustos for kindly providing

Anonymous (1994), Tecnología para la producción de pitahaya. INRA-CEE, p. 70.

Butera D., Tesoriere L., Di Gaudio F., Bongiorno A., Allegra M., Pintaudi A. M., Kohen R., and Livrea M. A. (2002), Antioxidant activities of Sicilian prickly pear (Opuntia ficus indica) fruit extracts and reducing properties of its betalains: betanin and indicaxanthin. J. Agric. Food Chem. 50, 6895-6901.

Cháfer A., Fornari T., Stateva R. P., Berna A., and García-Reverter J. (2007), Solubility of the natural antioxidant gallic acid in supercritical $\mathrm{CO}_{2}+$ ethanol as cosolvent. J. Chem. Eng. Data 52, 116-121.

Chun O. K., Kim D.-O., Smith N., Schroeder D., Kang H. G., and Lee C. Y. (2005), Daily consumption of phenolics and total antioxidant capacity from fruit and vegetables in the American diet. J. Sci. Food Agric. 85, 1715-1724.

Esquivel P., Stintzing F. C., and Carle R. (2007), Comparison of morphological and chemical fruit traits from different pitaya genotypes (Hylocereus sp.) grown in Costa Rica. J. Appl. Bot. Food Qual. 81, $7-14$.

Herbach K. M., Stintzing F. C., and Carle R. (2004), Thermal degradation of betacyanins in juices from purple pitaya (Hylocereus polyrhizus [Weber] Britton \& Rose) monitored by high-performance liquid chromatography-tandem mass spectrometric analyses. Eur. Food. Res. Technol. 219, 377-385.

Herbach K. M., Stintzing F. C., and Carle R. (2005), Identification of heat-induced degradation products from purified betanin, phyllocactin and hylocerenin by high-performance liquid chromatography/electrospray ionisation mass spectrometry. Rapid Comm. Mass Spectr. 19, 2603-2616; 20, 1822.

Kammerer D., Claus A., Schieber A., and Carle R. (2004), Polyphenol screening of pomace from red and white grapes varieties (Vitis vinifera L.) by HPLCDAD-MS/MS. J. Agric. Food Chem. 52, 4360-4367.

Kanner K., Harel S., and Granit R. (2001), Betalains A new class of dietary cationized antioxidants. J. Agric. Food Chem. 49, 5178-5185.

Kobayashi N., Schmidt J., Wray V., and Schliemann W. (2001), Formation and occurrence of dopamine-derived betacyanins. Phytochemistry 56, 429-436.

Kugler F., Graneis S., Schreiter P. P. Y., Stintzing F. C., and Carle R. (2006), Determination of free amino compounds in betalainic fruits and vegetables by gas the fruits used in this investigation and for their support during the field work. This investigation was partly funded by the University of Costa Rica (project number VI-734-A5-029). P. E. received a short-time scholarship from the German Academic Exchange Service (DAAD) to accomplish this study.

chromatography with flame ionisation and mass spectrometry detection. J. Agric. Food Chem. 54, 43114318.

Kugler F., Stintzing F. C., and Carle R. (2007), Characterisation of betalain patterns of differently coloured inflorescences from Gomphrena globosa L. and Bougainvillea sp. by HPLC-DAD-ESI-MS ${ }^{n}$. Anal. Bioanal. Chem. 387, 637-648.

Kujala T., Klika K., Ovcharenko V., Loponen J., Vienola M., and Pihlaja K. (2001), 5,5',6,6' -Tetrahydroxy-3,3' biindolyl from beetroot (Beta vulgaris) peel extract. Z. Naturforsch. 56c, 714-718.

Kujala T. S., Vienola M. S., Klika K. D., Loponen J. M., and Pihlaja K. (2002), Betalain and phenolic compositions of four beetroot (Beta vulgaris) cultivars. Eur. Food Res. Technol. 214, 505-510.

Mahattanatawee K., Manthey J. A., Luzio G., Talcott S. T., Goodner K., and Baldwin E. A. (2006), Total antioxidant activity and fiber content of select Floridagrown tropical fruits. J. Agric. Food Chem. 54, $7355-7363$.

Mizrahi Y. and Nerd A. (1999), Climbing and columnar cacti: New arid land fruit crops. In: Perspectives on New Crops and New Uses (Janick J., ed.). American Society for Horticultural Science, Alexandria, Virginia, pp. 358-366.

Nerd A. and Mizrahi Y. (1997), Reproductive biology of cactus fruit crops. Hort. Rev. 18, 322-346.

Priyadarsini K. I., Khopde S. M., Kumar S. S., and Mohan H. (2002), Free radical studies of ellagic acid, a natural phenolic antioxidant. J. Agric. Food Chem. 50, 2200-2206.

Rommel A. and Wrolstad R. E. (1993), Composition of flavonols in red raspberry juice as influenced by cultivar, processing, and environmental factors. J. Agric. Food Chem. 41, 1941-1950.

Sakakibara H., Honda Y., Nakagawa S., Ashida H., and Kanazawa K. (2003), Simultaneous determination of all polyphenols in vegetables, fruits and teas. J. Agric. Food Chem. 51, 571-581.

Sánchez-Moreno C. (2002), Review: Methods used to evaluate the free radical scavenging activity in foods and biological systems. Food Sci. Tech. Int. 8, 121137.

Singleton V. L., Orthofer R., and Lamuela-Raventós R. M. (1999), Analysis of total phenols and other oxi- 
dation substrates and antioxidants by means of FolinCiocalteu reagent. Meth. Enzymol. 299, 152-178.

Stintzing F. C., Schieber A., and Carle R. (2002), Betacyanins in fruits from red-purple pitaya, Hylocereus polyrhizus (Weber) Britton \& Rose. Food Chem. 77, 101-106, 517.

Stintzing F. C., Schieber A., and Carle R. (2003), Evaluation of colour properties and chemical quality parameters of cactus juices. Eur. Food Res. Technol. 216, $303-311$.

Stintzing F. C., Conrad J., Klaiber I., Beifuss U., and Carle R. (2004), Structural investigations on betacyanin pigments by LC NMR and 2D NMR spectroscopy. Phytochemistry 65, 415-422.

Stintzing F. C., Herbach K. M., Moßhammer M. R., Carle R., Yi W., Sellapan S., Akoh C. C., Bunch R., and Felker P. (2005), Colour, betalain pattern and antioxidant properties of cactus pear (Opuntia sp.) clones. J. Agric. Food Chem. 53, 442-451.

Strack D., Vogt T., and Schliemann W. (2003), Recent advances in betalain research. Phytochemistry 62, $247-269$.

Vaillant F., Perez A., Davila I., Dornier M., and Reynes M. (2005), Colorant and antioxidant properties of redpurple pitahaya (Hylocereus sp.). Fruits 60, 1-10.
Van den Berg R., Haenen G. R. M. M., van den Berg H., van der Vijgh W., and Bast A. (2000), The predictive value of the antioxidant capacity of structurally related flavonoids using the Trolox equivalent antioxidant capacity (TEAC) assay. Food Chem. 70, 391-395.

Wang K. J., Yang C. R., and Zhang Y. J. (2007), Phenolic antioxidants from Chinese toon (fresh young leaves and shoots of Toona sinensis). Food Chem. 101, $365-371$.

Wu L., Hsu H. W., Chen Y. Z., Chiu C. C., Lin Y. I., and Ho J. A. (2006), Antioxidant and antiproliferative activities of red pitaya. Food Chem. 95, 319-327.

Wybraniec S. (2005), Formation of decarboxylated betacyanins in heated purified betacyanin fractions from red beet root (Beta vulgaris L.) monitored by LC-MS/ MS. J. Agric. Food Chem. 53, 3483-3487.

Wybraniec S. and Mizrahi Y. (2005), Generation of decarboxylated and dehydrogenated betacyanins in thermally purified fruit extract from purple pitaya (Hylocereus polyrhizus) monitored by LC-MS/MS. J. Agric. Food Chem. 53, 6704-6712.

Wybraniec S., Nowak-Wydra B., Mitka K., Kowalski P., and Mizrahi Y. (2007), Minor betalains in fruits of $\mathrm{Hy}$ locereus species. Phytochemistry 68, 251-259. 